\title{
Prácticas docentes para promover la autorregulación del aprendizaje durante la pandemia COVID-19: escalas de medición y modelo predictivo
}

\author{
Fabiola Sáez-Delgado ${ }^{{ }^{*}}$, Yaranay López-Angulo ${ }^{2,3}$, Javier Mella-Norambuena ${ }^{4}$ y Daniel Casanova ${ }^{5}$ \\ (1) Universidad Católica de la Santísima Concepción, Centro de Investigación en Educación y Desarrollo y Facultad de \\ Educación, Departamento Fundamentos de la Pedagogía, Concepción-Chile (Correo-e: fsaez@ucsc.cl) \\ (2) Universidad Santo Tomás, Fac. de Ciencias Sociales y Comunicaciones, Escuela de Psicología, Concepción, Chile. \\ (3) Departamento de Psicología, Facultad de Ciencias Sociales, Universidad de Concepción, Concepción-Chile. \\ (Correo-e: yara13190@gmail.com) \\ (4) Universidad Técnica Federico Santa María, Departamento de Ciencias, Concepción, Chile. \\ (Correo-e: javier.mellan@usm.cl) \\ (5) Dirección de Docencia, Universidad Católica de la Santísima Concepción, Chile. (Correo-e: casanova@ucsc.cl)
}

* Autor a quien debe ser dirigida la correspondência.

Recibido Jun. 24, 2021; Aceptado Ago. 19, 2021; Versión final Sep. 13, 2021, Publicado Feb. 2022

\begin{abstract}
Resumen
Los objetivos principales de esta investigación fueron confirmar la estructura factorial de escalas sobre variables docentes para la promoción de la autorregulación del aprendizaje en estudiantes y estimar un modelo predictivo de las prácticas docentes durante la pandemia COVID-19. El método consideró un diseño psicométrico y predictivo para cada fase. Participaron 765 docentes de seis universidades de Chile. Los resultados mostraron cuatro escalas (creencias, autoeficacia, conocimiento y prácticas docentes) con adecuadas propiedades psicométricas. Se detectaron efectos indirectos de las variables "conocimientos de aprendizaje autorregulado" y "autoeficacia para la promoción de aprendizaje autorregulado" sobre la variable "prácticas docentes de promoción del aprendizaje autorregulado", siendo esta una mediación de tipo parcial. El modelo de mediación estimado predice en un 33.7\% las prácticas docentes para la promoción de aprendizaje autorregulado. En conclusión, se disponen de escalas válidas y confiables y de un modelo predictivo con adecuado ajuste para comprender la promoción de la autorregulación de aprendizaje por parte de docentes universitarios.
\end{abstract}

Palabras clave: prácticas docentes; autorregulación; aprendizaje; educación superior; modelo predictivo; COVID-19

\section{Teaching practices for enhancing self-regulated learning during the COVID-19 pandemic: measuring scales and predictive model}

\begin{abstract}
The main objectives of this research study were to examine the factorial structure of teaching-variable scales to enhance self-regulated learning in students and to estimate a predictive model for teaching practices during the COVID-19 pandemic. The methodology applied contained a psychometric and a predictive design for each phase of the study. A total of 765 professors from six Chilean universities were surveyed. The results showed four scales (beliefs, self-efficacy, knowledge, and teaching practices) with adequate psychometric properties. The variables "self-regulated learning knowledge" and "self-efficacy for promoting self-regulated learning" had indirect effects on the variable "teaching practices for promoting self-regulated learning", which was a partial mediation type. The estimated mediation model accurately predicted $33.7 \%$ of the teaching practices used for boosting self-regulated learning. In conclusion, the present study developed valid and reliable scales along with a predictive model with adequate adjustment that served to improve the understanding of self-regulated learning enhanced by university professors.
\end{abstract}




\section{INTRODUCCIÓN}

La Covid-19, establecida como pandemia por la Organización Mundial de la Salud el 11 de marzo de 2020 (OMS, 2020), provocó que los gobiernos aplicaran distintas medidas para salvaguardar la vida humana; el distanciamiento físico generó que muchos sectores, incluido el sector educativo, tuvieran que detener sus actividades temporalmente. Así las universidades tuvieron que cambiar la educación presencial hacia una forzada e improvisada educación en línea denominada Enseñanza Remota de Emergencia (en adelante ERE) (Iglesias-Pradas et al., 2021).

A diferencia de los programas educativos en modalidad online, cuyas experiencias de aprendizaje fueron planificadas y diseñadas desde el principio para estar en línea; la ERE es un modo alternativo de enseñanza ante circunstancias de crisis, repentino y temporal en los procesos de formación académica. Implica el uso a distancia de soluciones de enseñanza para la educación que anteriormente se impartían presencialmente, y cuyo objetivo principal no es recrear un ecosistema educativo sólido, sino proporcionar temporalmente los recursos educativos necesarios de una manera ágil y confiable (Iglesias-Pradas et al., 2021). La ERE requirió un esfuerzo de docentes y sus estudiantes para continuar con el proceso educativo. Los profesores tuvieron que desarrollar nuevas prácticas para responder a las nuevas exigencias y garantizar que las clases pudieran continuar; por su parte los estudiantes ahora más que nunca, han requerido gestionar sus recursos personales para la preparación del estudio y logro de sus aprendizajes, es decir, han necesitado autorregularse (Harahap y Harahap, 2020; Johari y Syaveny, 2020; Nepal y Kumar, 2021).

La autorregulación del aprendizaje (en adelante ARA) se destaca como una competencia clave para el éxito de los estudios en la Educación Superior, y adquiere relevancia adicional cuando los estudiantes enfrentan una situación de aprendizaje por medio de la ERE con una estructura abruptamente diferente a la que estaban acostumbrados, teniendo que liderar con mayor eficacia su estudio y aprendizaje autónomo (Pelikan et al., 2021). La ARA se enmarca en la teoría del aprendizaje cognitivo social (Schunk, 1990). Pintrich (2000), es uno de los autores que continúa siendo importante en el campo de la ARA, ya que contribuyó a aclarar su marco conceptual estudiando su relación con la motivación, y enfatizó en las diferencias entre metacognición y autorregulación. Así, la ARA se ha sido definida en la literatura especializada como un proceso dinámico de tres fases cíclicas (disposición, desempeño y evaluación), donde el estudiante reflexiona y dirige su actuación (planifica, monitorea y adapta emociones, cogniciones y comportamientos) con sentido de autodeterminación de su estudio y aprendizaje para lograr objetivos académicos (Pintrich, et., al. 2000, Sáez-Delgado et al., 2020b; Sáez-Delgado et al., 2021; Zimmerman, 2000).

Las estrategias de ARA desplegadas en la fase de disposición (establecimiento de objetivos y planificación) permiten a los estudiantes establecer metas positivas, pequeñas (factibles), precisas (posibles de medir) y personales (tienen un sentido propio); las estrategias desplegadas en la fase de desempeño permiten a los estudiantes monitorear su proceso de estudio y aprendizaje; las estrategias desplegadas en la fase de evaluación permite a los estudiantes observar el resultado de su desempeño y determinar los ajustes necesarios para mejorar en una futura actuación (Sáez-Delgado et al., 2020a; Sáez-Delgado et al., 2018).

La mayoría de los estudiantes no se encuentran adecuadamente preparados para lo que se requiere de ellos, ya que no son autorregulados (Schober et al., 2015). Esta falta de competencias de autorregulación para afrontar el aprendizaje es un factor principal del fracaso académico en la Educación Superior (Hoops et al., 2016; SáezDelgado et al., 2020b). Con el contexto actual de pandemia, este problema se acrecentó revelando grandes dificultades en los procesos autorregulatorios de los estudiantes; una investigación reciente evidenció que sólo un $17,5 \%$ de los estudiantes fueron clasificados en la categoría de nivel alto de autorregulación lo que revela una gran necesidad de adaptación para el aprendizaje de los estudiantes durante la pandemia y la urgente necesidad que se fomente su autoaprendizaje (aprendizaje autorregulado) (Harahap y Harahap, 2020). Otro estudio mostró niveles regulares de autorregulación y sobre la base de sus hallazgos concluyó que los estudiantes tienen distintos niveles en sus procesos autorregulatorios y no todos están listos para afrontar la ERE (Johari y Syaveny, 2020). Por su parte un estudio con profesores reveló que los estudiantes evidencian una falta de habilidades para el aprendizaje continuo y autónomo, es decir, niveles insuficientes de ARA durante la pandemia de COVID-19 para continuar con una respuesta eficaz en sus aprendizajes (Nepal y Kumar, 2021).

En Chile, un estudio en 240 universitarios de primer año en una universidad pública durante la emergencia sanitaria (Rivera y Millán, 2021), ha revelado que el estudiantado se muestra preocupado de la modalidad de ERE y sugieren que los profesores mejoren aspectos metodológicos de sus clases incorporando diferentes formas para aprender el contenido de la clase dando más instancias de participación e interacción. Así también, un estudio realizado en profesores universitarios de tres regiones de la Macro Zona Centro Sur de Chile (Maule, Ñuble y Bío Bío), sobre las concepciones docentes de enseñanza durante el contexto de pandemia, reveló, que en general, los académicos de las distintas regiones comparten similares ideas frente a este nuevo escenario, destacando que el aspecto emocional es uno de los elementos que ha sufrido mayor impacto durante esta crisis sanitaria afectando tanto al estudiantado como al profesorado, haciendo énfasis en las dificultades de enseñanza 
por la escasa interacción y dificultando la relación profesor estudiante. Por consiguiente, se demuestra con mayor fuerza la importancia del rol de los docentes ante situaciones extraordinarias para que sus estudiantes logren los aprendizajes y procesos académicos exitosos, aún en escenarios adversos.

A nivel internacional, resultados de una investigación durante la emergencia sanitaria evidenció que aquellos docentes que guían su enseñanza por protocolos basados en el aprendizaje autorregulado logran mejores resultados académicos en sus estudiantes (Cai et al., 2020). Por tal motivo, el énfasis se ha puesto en la necesidad de que los docentes fomenten en sus estudiantes esta competencia clave para el éxito académico (Sava et al., 2020) y, aunque gran parte de los docentes está de acuerdo en que enseñar a los estudiantes a ser más autorregulados en sus clases sería ideal; en la práctica existen desafíos importantes para lograrlo (Dignath y Veenman, 2021). La instrucción que realizan los docentes para el fomento de la ARA en las dinámicas propias del desarrollo de las clases es poco frecuente y, cuando se realiza, es poco eficaz. Los estudios han demostrado lagunas en el conocimiento y las creencias epistémicas de los docentes sobre ARA (Barr y Askell-Williams, 2020). Además, históricamente en los docentes universitarios se han detectado carencias en las prácticas de promoción de estrategias de ARA (Peel, 2020).

En el contexto de Educación Superior, se han identificado en los profesores creencias epistemológicas inadecuadas, bajos niveles de autoeficacia y conocimiento sobre la promoción de ARA, y consecuentemente un bajo nivel de prácticas de fomento de la ARA en sus estudiantes (Daura, 2017). Sin embargo, cuando los docentes informan mayores creencias de que los estudiantes pueden beneficiarse de ARA, cuanto más conocimiento tengan sobre cómo promover la ARA y cuanto más capaces se sienten de instruir estrategias de ARA; más informan las prácticas de promoción de la ARA cuando enseñan y ofrecen más situaciones de aprendizaje que permiten su desarrollo (Dignath, 2016).

Las escasas investigaciones disponibles revelan que pocos profesores poseen creencias sobre los beneficios de fomentar en sus estudiantes la ARA (Dignath y Veenman, 2021). Para que los docentes fomenten explícitamente la aplicación de estrategias de ARA, se requiere que crean en la importancia de esta competencia para sus estudiantes (Barr y Askell-Williams, 2020). Docentes con conocimiento de contenido pedagógico de alta calidad sobre ARA emplearían instrucción de estrategia explícita, como nombrar, explicar, modelar, discutir, estimular y practicar varias estrategias de ARA (Kistner et al., 2015). Sin embargo, los estudios han demostrado que los docentes carecen de conocimiento pedagógico sobre ARA (Spruce y Bol, 2015). De igual modo, la autoeficacia de los docentes para promover la ARA se ha identificado en niveles bajos, los docentes no se perciben capaces de instruir a sus estudiantes esta competencia y consecuentemente de promoverla (De Smul et al., 2018).

Las investigaciones han señalado dentro de las tres principales variables con valor predictivo de las prácticas docentes de promoción de la ARA en estudiantes a: (1) las creencias docentes de ARA (Kistner et. al., 2015), (2) la autoeficacia docente para promoción de ARA (Dignath, 2016) y (3) conocimiento docente de ARA (Merchan y Hernández, 2018). Existen algunos antecedentes de modelos donde se han integrado estas variables como predictores de las prácticas docentes de ARA (Barr y Askell-Williams, 2020; Dignath, 2016; Hoops et al., 2016). Sin embargo, son escasas las propuestas de modelos en Latinoamérica que permitan explicar cuáles son las variables que predicen las prácticas de los docentes universitarios para promover la ARA en el actual contexto de ERE. Además, existe un vacío investigativo acerca de la disposición de instrumentos válidos y confiables para medir variables docentes que se advierten como claves en este fenómeno.

Actualmente predominan diversos métodos de medición de la ARA en estudiantes (Sáez-Delgado et al., 2021), sin embargo, no es el caso para los docentes, más aún considerando la complejidad del fenómeno y su característica multidimensional. Por tales razones los objetivos establecidos en este estudio son: (1) Confirmar la estructura factorial de las escalas sobre variables docentes para la promoción de autorregulación del aprendizaje en estudiantes universitarios; y (2) estimar un modelo predictivo de las prácticas docentes de promoción de la autorregulación en estudiantes universitarios a partir de las creencias de ARA, creencias de autoeficacia para promover ARA y conocimientos de ARA que poseen los profesores durante la ERE.

\section{MÉTODO}

El estudio se desarrolló en dos fases consistentes en un diseño instrumental para el primer objetivo y un diseño predictivo para el segundo y principal (Ato et al., 2013). El modelo definido preliminarmente a partir de los antecedentes teóricos y empíricos presentados consiste en el valor predictivo de las prácticas de promoción de ARA a partir de: (1) las creencias docentes sobre ARA (por ejemplo, si es útil o no para la enseñanza), (2) los conocimientos que posea sobre esta competencia (por ejemplo, conoce diferentes prácticas de instrucción) y (3) las creencias de autoeficacia sobre la promoción de la ARA (es decir, en qué medida se siente capaz de implementar formas de instrucción de ARA). Se puede definir, por tanto, que el comportamiento de los docentes asociado a las prácticas de promoción de la ARA que implementan o no en la cotidianidad de sus clases con el estudiantado, está determinado por las creencias, conocimientos y autoeficacia que tienen acerca de los procesos de autorregulación del aprendizaje. 


\section{Participantes}

La muestra de esta investigación fueron 765 docentes universitarios de seis universidades pertenecientes al Consejo de Rectores de Chile. Mujeres fueron $367(48 \%)$ con una edad promedio de 45 años (DE = 6.2) y $398(52 \%)$ hombres con una edad promedio de 47 años $(D E=7.4)$. El acceso fue por conveniencia durante el primer semestre de 2020. Los docentes pertenecían a distintas facultades. Facultad de Ciencias Económicas y Administrativas $n=70$ (9.15\%), Facultad de Ciencias Físicas y Matemáticas $n=127$ (16.55\%), Facultad de Ciencias Jurídicas $n=32(4.23 \%)$, Facultad de Ciencias Sociales $n=75(9.86 \%)$, Facultad de Educación $n=156(20.42 \%)$, Facultad de Humanidades y Arte $n=30(3.87 \%)$, Facultad de Ingeniería $n=102$ (13.38\%), Facultad de Medicina $n=127$ (16.55\%) y de otras Facultades $n=46$ (5.99\%).

Para los objetivos de este estudio se dividió el total de la muestra en dos grupos, quedando 382 participantes para el desarrollo del objetivo 1 de este estudio correspondiente al estudio de la estructura factorial de las escalas; y 383 para el desarrollo del objetivo 2 correspondiente al modelo predictivo. El método de división de la muestra para responder a cada objetivo fue aleatorio, donde se utilizó como herramienta el comando sample() del Software R considerando los ID (número de identificación de cada participante) e indicando una semilla (punto de partida) para que se pudiera ejecutar la función de aleatorización. Este procedimiento permitió la asignación al azar de cada sujeto a una etapa del estudio.

\section{Instrumentos de medida}

Para la medición de variables docentes promotoras de la ARA, los autores de esta investigación construyeron y validaron las escalas. Su diseño y validación inicial se realizaron en el contexto del proyecto de investigación de Sáez-Delgado (2020). Estas escalas tuvieron como referente teórico para su construcción las investigaciones de (De Smul et al., 2018; Dignath y Veenman, 2021; Spruce y Bol, 2015), el modelo de Zimmerman (2000) y la experiencia en desarrollo de instrumentos de autorregulación diseñados para estudiantes (Sáez-Delgado et al., 2021;) y profesores (Sáez-Delgado et al., 2020a). Las características de las escalas diseñadas y validadas en profesores universitarios chilenos en el proyecto de Sáez-Delgado (2020), contaban con validación de 5 jueces expertos para mostrar evidencia de validez de contenido (3 nacionales y dos internacionales), por procesos de entrevista cognitiva para mostrar evidencia del formato de respuesta ( 5 docentes) y por análisis factorial exploratorio para mostrar evidencia inicial de su estructura dimensional. El tiempo de respuesta promedio de los profesores fue de 10 minutos.

Las escalas quedaron conformadas de la siguiente manera: (1) Creencias docentes de ARA, mide las creencias de los docentes acerca de los procesos de ARA en estudiantes, escala unidimensional, consta de 3 ítems y se responde en una escala tipo Likert de diez puntos que va desde 0 (No creo que sea así), hasta 10 (Creo con mucha certeza que es así); (2) Autoeficacia docente para la Promoción de ARA, mide las creencias de los profesores sobre su propia capacidad para promover en sus estudiantes la autorregulación del aprendizaje, escala unidimensional, consta de 3 ítems y se responde en una escala tipo Likert de diez puntos que va desde 0 (No creo que lo pueda hacer), hasta 10 (Creo con mucha seguridad que lo puedo hacer); (3) Conocimiento de ARA, mide cuánto conoce el docente sobre el proceso de ARA, tiene en total 14 ítems, es una escala de tres dimensiones, la primera corresponde a conocimiento sobre estrategias de ARA y tiene 4 ítems, la segunda corresponde a conocimiento de formas de instrucción de ARA y tiene 3 ítems y la tercera corresponde a conocimiento sobre creencias de autoeficacia y atribución causal y tiene 3 ítems, se responde en una escala tipo Likert de siete puntos que va desde 1 (no conozco nada) hasta 10 (conozco todo); (4) Prácticas docentes de promoción de ARA, mide la frecuencia con que los docentes promueven el aprendizaje autorregulado en sus estudiantes, escala unidimensional tiene 4 ítems y se responde en una escala tipo Likert de siete puntos que va desde 1 (nunca) hasta 10 (siempre). En total, las 4 escalas quedaron compuestas por 22 ítems y en su conjunto miden las variables docentes promotoras de la autorregulación del aprendizaje en estudiantes universitarios (Ver Tabla 1).

\section{Procedimiento}

El 27 de julio de 2020 se inició la recolección de los datos por medio de una encuesta en línea usando la herramienta Surveymonkey. La primera sección de la encuesta presentaba un consentimiento informado donde los docentes invitados a ser parte del estudio seleccionaban si aceptaban o no participar. A quienes aceptaban participar, se les desplegaba la siguiente sección que incluía las 4 escalas de esta investigación. El contacto con los docentes fue por medio de correo electrónico, donde se les invitaba a participar del estudio y donde se especificaba el link de acceso para responder la encuesta. Se pidió colaboración de difusión a las redes de universidades en Chile. La recolección de datos se prolongó dos meses se procedió a (julio y agosto de 2020). Estos meses correspondieron al inicio del segundo semestre académico del año 2020 en Chile. Este estudio fue aprobado por el Comité de ética de la Universidad Católica de la Santísima Concepción, Chile. 
Tabla 1. Escalas para medir variables docentes de promoción de la autorregulación del aprendizaje en estudiantes universitarios

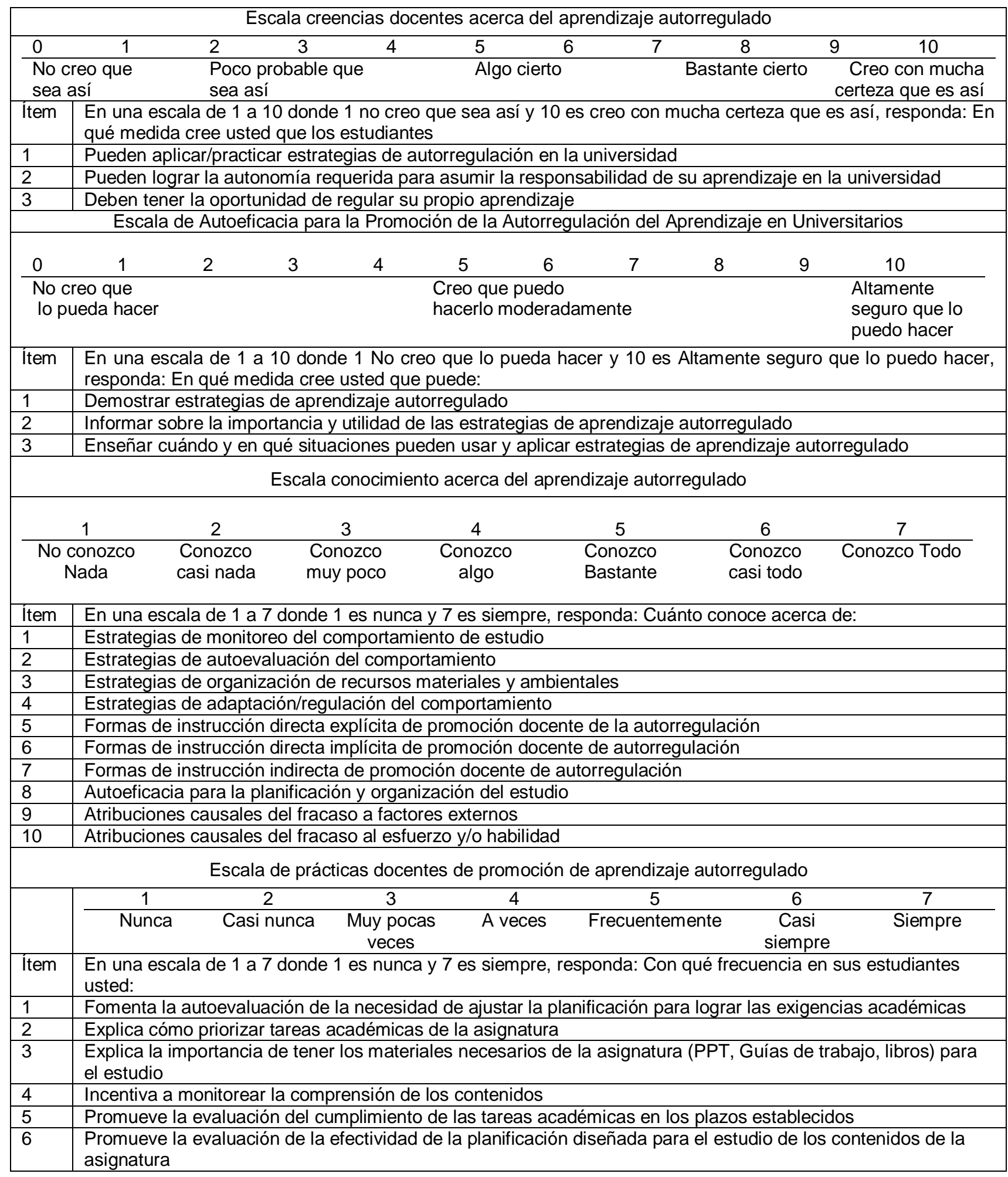

\section{Análisis de datos}

Para dar cumplimiento al primer objetivo de este estudio, se desarrolló análisis factorial confirmatorio (AFC) a las 4 escalas, para el segundo objetivo se realizaron análisis de mediación. Para evaluar la bondad de ajuste se seleccionaron los siguientes índices: raíz cuadrada media de error de aproximación (RMSEA) con un intervalo de confianza al 90 \% (I.C.), el índice de ajuste comparativo (CFI), índice de Tucker-Lewis (TLI), definiendo los siguientes criterios: (a) CFI o TLI sobre .90, (b) RMSEA menores a .70 (Wang y Wang, 2019). Todos los análisis se realizaron en el software Mplus Versión 8.6. 


\section{RESULTADOS}

La fase 1 consistió en la estimación de las propiedades psicométricas de la batería para la medición de las variables docentes promotoras de la autorregulación del aprendizaje. En la Tabla 2, se describen los resultados de los análisis factoriales confirmatorios, donde: $X^{2}=$ chi cuadrado; df = grados de libertad del modelo; RMSEA = Error Medio Cuadrático de Aproximación; $(90 \% \mathrm{Cl})=$ Intervalo de confianza del 90\% para RMSEA; $\mathrm{TLI}=$ índice de Tucker-Lewis; $\mathrm{CFI}=$ Índice de Ajuste Comparativo; ${ }^{*} \mathrm{p}<.05$. La confiabilidad se estimó con los índices alpha $(\boldsymbol{\alpha})$ y omega $(\Omega)$. La escala de creencias docentes de ARA, conocimiento de ARA, prácticas docentes de promoción de ARA y autoeficacia para la promoción de ARA presentaron ajustes adecuados.

Tabla 2. Estimaciones del Modelo de AFC

\begin{tabular}{|l|c|c|c|c|c|c|c|c|c|}
\hline \multicolumn{1}{|c|}{ Escalas } & $\mathrm{X}^{2}$ & Df & RMSEA & $(90 \%$ Cl $)$ & SRMR & CFI & TLI & $\alpha$ & $\Omega$ \\
\hline $\begin{array}{l}\text { Creencias docentes acerca de } \\
\text { ARA }\end{array}$ & $.000^{*}$ & 0 & .000 & $.000-.000$ & .000 & 1.000 & 1.000 & .922 & .925 \\
\hline $\begin{array}{l}\text { Conocimiento docente acerca de } \\
\text { ARA }\end{array}$ & $125.627^{*}$ & 30 & .065 & $.054-.077$ & .021 & .971 & .956 & .957 & .957 \\
\hline $\begin{array}{l}\text { Prácticas docentes de } \\
\text { promoción de ARA }\end{array}$ & $22.053^{*}$ & 9 & .065 & $031-.100$ & .037 & .968 & .947 & .822 & .825 \\
\hline $\begin{array}{l}\text { Autoeficacia para la promoción } \\
\text { de ARA }\end{array}$ & $.000^{*}$ & 0 & .000 & $.000-.000$ & .000 & 1.000 & 1.000 & .820 & .837 \\
\hline
\end{tabular}

Los resultados del análisis presentado permiten concluir que el modelo de medida posee índices de ajuste adecuados para proseguir con la siguiente fase. La fase 2 consistió en el modelo predictivo de mediación. Previo a la estimación del modelo predictivo se presentan los estadísticos descriptivos y correlaciones, estas últimas son un paso imprescindible antes de la estimación de cualquier análisis de modelación. En la Tabla 3 , se presentan los análisis descriptivos; donde se puede observar que los docentes presentan puntuaciones medias respecto a sus creencias de aprendizaje autorregulado $(M=6.21, D E=2.794)$; puntuaciones bajas de conocimiento acerca de aprendizaje autorregulado $(M=2.96, D E=1.271)$; puntuaciones medias de prácticas de promoción de la ARA $(M=4.63, D E=.862)$; y puntuaciones medias de autoeficacia para la promoción de la ARA $(M=7.44, D E=1.859)$. Se observa en la Tabla 3 que las variables tienen correlaciones significativas, siendo la más fuerte entre prácticas docentes de promoción de aprendizaje autorregulado y autoeficacia para la promoción de aprendizaje autorregulado $(r=.474, \mathrm{p}<.001)$. Si bien las variables tienen relación, el coeficiente no es extremadamente alto como para indicar multicolinealidad (Field, 2009).

Tabla 3. Estadísticos descriptivos y correlaciones entre las escalas

\begin{tabular}{|l|c|c|c|c|c|c|}
\hline \multicolumn{1}{|c|}{ Variables } & $\mathrm{M}$ & $\mathrm{DE}$ & $(1)$ & $(2)$ & $(3)$ & (4) \\
\hline (1) Creencias docentes acerca del aprendizaje autorregulado & 6.63 & 2.794 & 1 & $.378^{* *}$ & $.252^{* *}$ & $.424^{* *}$ \\
\hline (2) Conocimientos acerca del aprendizaje autorregulado & 2.96 & 1.271 & & 1 & $.328^{* *}$ & $.436^{* *}$ \\
\hline (3) Prácticas docentes de promoción de aprendizaje autorregulado & 4.63 & .862 & & & 1 & $.474^{* *}$ \\
\hline (4) Autoeficacia para la promoción de aprendizaje autorregulado & 7.44 & 1.859 & & & & 1 \\
\hline
\end{tabular}

Los efectos totales indirectos de la variable creencias docentes de aprendizaje autorregulado sobre la variable prácticas docentes de promoción de aprendizaje autorregulado fueron estadísticamente significativos $(\beta=$ $.313 ; p<.001)$. Como consecuencia de lo anterior, se detectaron efectos indirectos específicos de las variables: (1) conocimientos de aprendizaje autorregulado $(\beta=.083 ; p<.001)$ y (2) autoeficacia para la promoción de aprendizaje autorregulado $(\beta=.230, \mathrm{p}<.001)$ sobre la variable práctica docentes de promoción de aprendizaje autorregulado, siendo esta una mediación de tipo parcial. El modelo predice un $33.7 \%$ de la variabilidad de las prácticas docentes de promoción de aprendizaje autorregulado.

\section{DISCUSIÓN}

La primera fase de esta investigación tuvo como propósito confirmar la estructura factorial de escalas sobre variables docentes para la promoción de ARA en universitarios. Los resultados obtenidos indicaron que las escalas presentan propiedades psicométricas adecuadas para ser utilizadas en docentes chilenos. Estas cuatro escalas compuestas por 22 ítems miden las variables docentes relevantes según la literatura e implicadas en la promoción de la ARA en universitarios. La condición de especificidad de cada escala representa la complejidad del fenómeno que se pretende medir. 

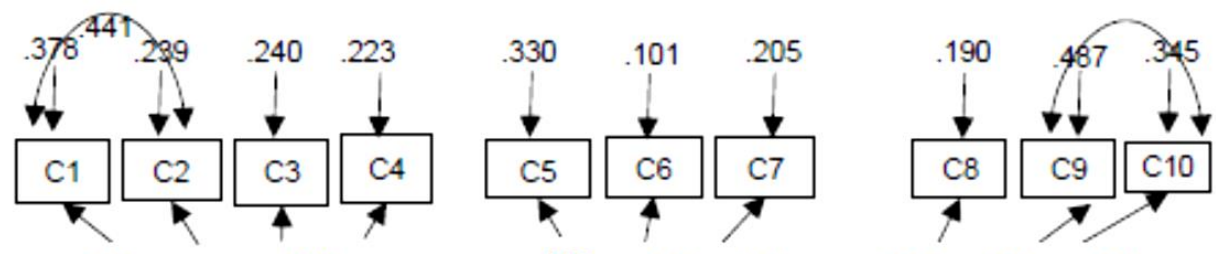

.789

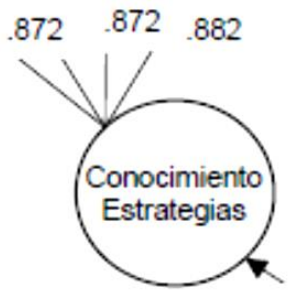

.906

$.948 \quad .891$

.900

.819

.809
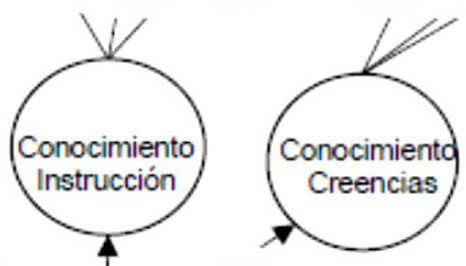

.972

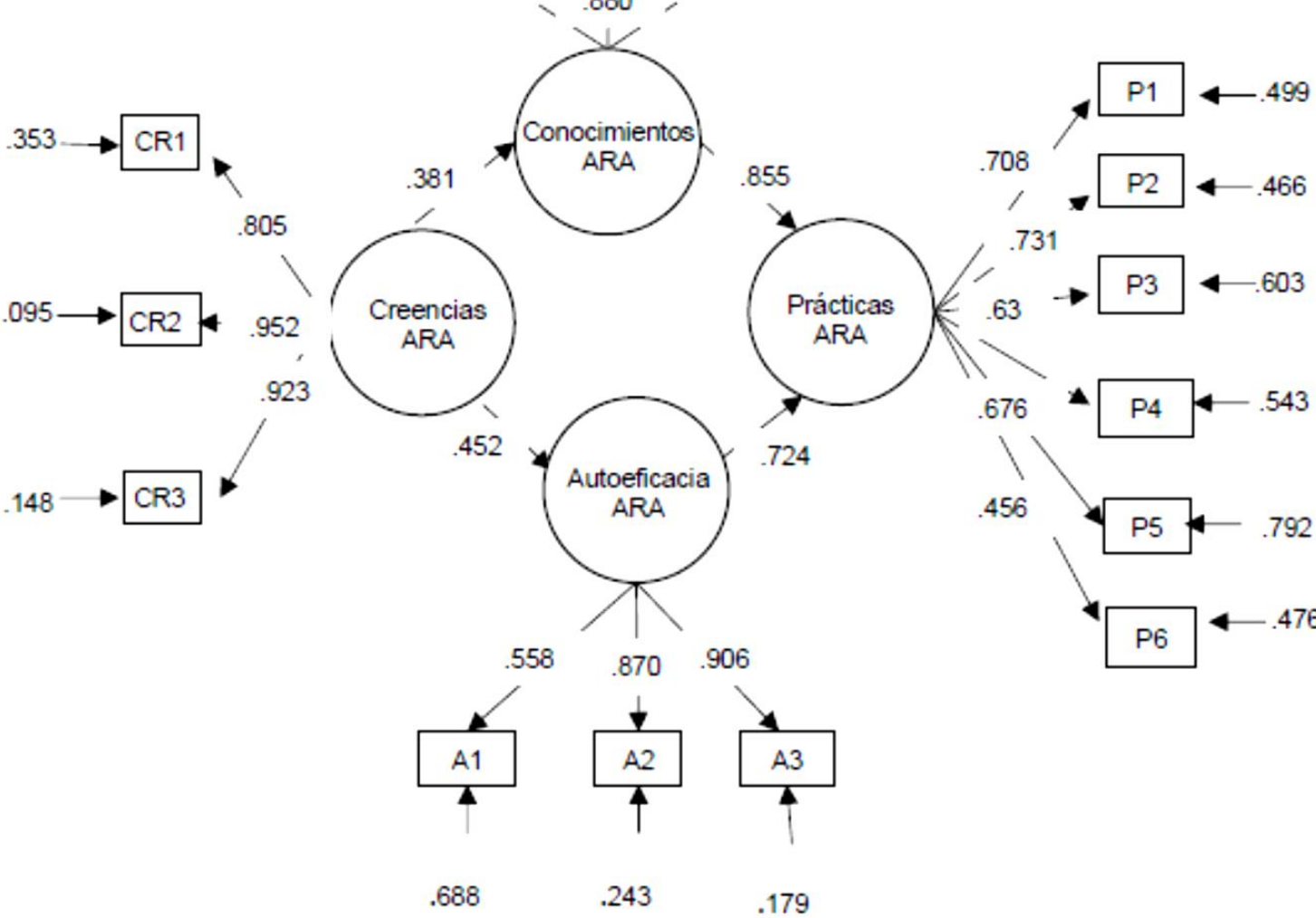

Fig. 1: Modelo predictivo de mediación de las prácticas docentes para la promoción de la ARA

Las escalas cuentan con las siguientes fortalezas: (1) han sido validadas en contexto latinoamericano y se encuentran en idioma español; (2) fueron validadas para su aplicación en contexto online, (3) todas las escalas son independientes entre sí, lo que permite a los investigadores usar una sola escala si se requiere para cumplir con determinados propósitos investigativos, (4) permite medir el efecto de intervenciones que integran variables docentes para la promoción de ARA; (5) tienen sólida fundamentación tanto teórica como empírica; y (6) siguieron un procedimiento psicométrico riguroso que muestra evidencia de la estructura factorial, es decir, confirman su dimensionalidad cumpliendo con los estándares de calidad según las recomendaciones en esta área. Por tanto, la etapa psicométrica de este estudio responde a la brecha referida, a la inexistencia de instrumentos válidos y confiables para medir variables docentes promotoras de ARA en contextos universitarios en la región latinoamericana. Contar con este instrumento permitirá el desarrollo de nuevas investigaciones con distintos alcances descriptivos, correlacionales, predictivos y explicativos para profundizar en el fenómeno de acuerdo con las particularidades de cada contexto universitario. Estudios previos que han desarrollado escalas en el área de la ARA en estudiantes han llegado a resultados similares, proponiendo escalas específicas para medir el constructo considerando su complejidad (Sáez-Delgado et al., 2021).

Estos resultados también son relevantes para la implementación de intervenciones de calidad, las cuales requieren de dos aspectos críticos que fueron abordados de manera consecutiva en esta investigación. Por una parte, contar con instrumentos válidos y confiables para medir lo que se ha propuesto en la investigación y por 
otra, contar con suficiente evidencia de modelos predictivos que identifiquen las variables que mejor predicen el fenómeno que se pretende explicar. En relación con esta última idea, esta investigación aportó evidencia acerca de las variables predictoras de las prácticas docentes de promoción de la ARA en estudiantes universitarios, evidenciando la importancia que tienen las creencias de ARA, autoeficacia para promoverla y conocimientos de ARA durante la ERE.

Otro resultado relevante fue el modelo predictivo propuesto, este responde a la necesidad de identificar las creencias de los docentes sobre concepciones y conceptos inadecuados acerca de la promoción de la ARA que influencian sus percepciones y por tanto sus prácticas, las cuales son necesarias en el diseño de intervenciones (Dignath y Veenman, 2021). En este estudio el modelo de mediación estimado logró predecir un $33.7 \%$ las prácticas docentes para la promoción de aprendizaje autorregulado, lo cual es considerado en Ciencias Sociales como un poder predictivo grande $(\mathrm{R} 2>$.25). Este resultado es similar a un estudio desarrollado en Alemania con 140 profesores de primaria que incluyó predictores sobre creencias epistemológicas, autoeficacia y conocimiento en el modelo y explicó el $38 \%$ de la varianza referida a la promoción de la autorregulación auto declarada por parte de los profesores (Dignath, 2016).

En el contexto de Educación Superior, se han identificado bajos niveles de autoeficacia y conocimiento de docentes respecto de la ARA y consecuentemente bajos niveles en las prácticas de fomento de la ARA en sus estudiantes (Daura, 2017). Modelos precedentes han mostrado el impacto de las creencias epistemológicas de los docentes sobre el valor de ARA, apoyando el supuesto de congruencia entre creencias y prácticas (Dignath, 2016). Por tanto, la implementación de las prácticas de ARA será más exitosa si los docentes tienen creencias positivas en línea con los supuestos de ARA.

Este estudio muestra evidencia robusta para considerar la inclusión de creencias, autoeficacia, conocimiento y prácticas relacionadas a la ARA en un programa de entrenamiento a docentes que tenga por objetivo mejorar la promoción de la ARA. Sin embargo, es importante considerar el contexto específico donde se realizó la investigación para la pertinente generalización de los datos en profesores universitarios chilenos que desarrollan docencia durante la ERE. Por tanto, una limitación de este estudio es el escenario bajo el cual se aplicó, que implica tener cuidado al generalizar los resultados en tiempos de enseñanza tradicional.

En el ámbito universitario se requiere ofrecer programas efectivos a los docentes para guiarlos durante la promoción de la ARA, que consideren el cambio en las creencias y la autoeficacia, además de la formación en conocimiento y prácticas efectivas (Daura, 2017). Se requieren programas específicos que les ayuden a traducir la teoría a la práctica (Spruce y Bol, 2015). En definitiva, esta investigación aporta un valioso conocimiento y proporciona evidencia específica de qué variables considerar en programas de intervención docente, que busquen mejorar las prácticas para fomentar la ARA. Futuras investigaciones podrían considerar en su diseño e implementación los resultados de ambas etapas de este estudio. Se proporciona un modelo con base teórica y empírica concordante con revisiones exhaustivas de la literatura que concluyen que los cambios en las experiencias de los docentes, acerca del apoyo de la ARA en el aula se relacionan con sus creencias, y son la base latente para sus comportamientos y decisiones de instrucción (Barr y Askell-Williams, 2020; Dignath, 2016; Hoops et al., 2016).

Esta propuesta de investigación se focalizó en el vacío teórico y empírico constatado en la literatura sobre escalas válidas y confiables que midan prácticas docentes de promoción de la ARA y además un modelo predictivo que permite comprender qué variables influyen en los docentes universitarios para que estos promuevan la ARA en el estudiantado. Considerando que las competencias de ARA son fundamentales para la consecución del éxito académico y consecuentemente, apoyarían una mayor retención de los estudiantes especialmente en los primeros años de experiencia en Educación Superior. Por tanto, el valor de este trabajo proporciona a investigadores un modelo para basar el diseño de intervenciones dirigidas a las instituciones de educación superior que busquen la mejora de los procesos de enseñanza y aprendizaje, la calidad de la enseñanza, y disminución del abandono, desde el entrenamiento docente para implementar la ARA de manera intracurricular en sus programas de asignatura, cuya efectividad puede ser demostrada por instrumentos con evidencias de validez en Chile.

\section{CONCLUSIONES}

A partir de los resultados obtenidos y de su discusión, se pueden establecer las siguientes conclusiones: 1) Se dispone de escalas válidas y confiables para medir creencias, conocimientos, autoeficacia y prácticas de docentes asociados a la promoción de la ARA; 2 ) las variables conocimientos de ARA y autoeficacia de ARA son mediadoras en la relación de creencias y prácticas de ARA; (3) el modelo propuesto permite predecir las prácticas docentes para la promoción de aprendizaje autorregulado y especifica las variables que pueden ser consideradas en el diseño de intervenciones que buscan mejorar estas prácticas en los docentes. 


\section{AGRADECIMIENTOS}

Al Proyecto INDIN 02/2019, titulado "Validación de un instrumento sobre variables predictoras de los docentes universitarios en la promoción intracurricular del aprendizaje autorregulado en sus estudiantes" financiado por la Dirección de Investigación de la Universidad Católica de la Santísima Concepción. Al Proyecto FONDECYT de Iniciación №11201054 titulado "La relación recíproca entre la autorregulación del profesor y la autorregulación del aprendizaje y desempeño académico del estudiante. Un modelo explicativo en educación media", financiado por la Agencia Nacional de Investigación y Desarrollo de Chile (ANID).

\section{REFERENCIAS}

Ato, M., López, J., y Benavente, A., Un Sistema de Clasificación de los Diseños de Investigación en Psicología, An. de Psicol, ISSN: 0212-9728, 29(3), 1038-1059 (2013)

Barr, S., y Askell-Williams, H., Changes in Teachers' Epistemic Cognition about Self-regulated Learning as they Engaged in a Researcher-Facilitated Professional Learning Community, https://doi.org/10.1080/1359866X.2019.1599098, Asia-Pac J Teach Edu., 48(2), 187-212 (2020)

Cai, R., Wang, Q., Xu, J., y Zhou, L., Effectiveness of Students' Self-Regulated Learning During the COVID-19 Pandemic, https://doi.org/10.15354/si.20.ar011, Sci Insigt., 34(1), 175-182 (2020)

Daura, F., Aprendizaje Autorregulado e Intervenciones Docentes en la Universidad, https://doi.org/10.15517/revedu.v41i2.21396, Rev. Educ., 41(2), 1-19 (2017)

De Smul, M., Heirweg, S., y otros tres autores, How Competent Do Teachers feel Instructing Self-Regulated Learning Strategies? Development and Validation of the Teacher Self-Efficacy Scale to Implement Self-Regulated Learning, https://doi.org/10.1016/j.tate.2018.01.001, Teach Teach Educ., 71, 214-225 (2018)

Dignath, C., Which Components of Teacher Competence Determine Whether Teachers Enhance Self-Regulated Learning? Predicting Teachers' Self-Reported Promotion of Self-Regulated Learning by means of Teacher Beliefs, Knowledge, and Self-efficacy, Front Lear Res, ISSN: 2295-3159, 4(5), 83-105 (2016)

Dignath, C., y Veenman, M., The Role of Direct Strategy Instruction and Indirect Activation of Self-Regulated LearningEvidence from Classroom Observation Studies, https://doi.org/10.1007/s10648-020-09534-0, Educ Psychol Rev., 33(2), 489-533 (2021)

Harahap, A., y Harahap, S., Covid 19: Self-Regulated Learning Mahasiswa, J Pendidik Dan Kel, ISSN: 2686-2859, 10(1), 36-42 (2020)

Hoops, L., Yu, S., Wang, Q., y Hollyer, V., Investigating Postsecondary Self-Regulated Learning Instructional Practices: the Development of the Self-regulated Learning Observation Protocol, Inter Handb Teach Educ, ISSN: 1812-9129, 28(1), 75-93 (2016)

Iglesias-Pradas, S., Hernández-García, Á., Chaparro-Peláez, J., y Prieto, J., Emergency Remote Teaching and Students' Academic Performance in Higher Education During the COVID-19 Pandemic: A Case Study, https://doi.org/10.1016/j.chb.2021.106713, Comput. Hum. Behav., 119(1), 1-18 (2021)

Johari, I., y Syaveny, N., Students' Self-regulated on Online Learning During covid 19 Pandemic, J Asian Stud, ISSN: 2774-5554, 1(1), 1-8 (2020)

Kistner, S., Rakoczy, K., Otto, B., y Klieme, E., Teaching Learning Strategies: The Role of Instructional Context and Teacher Beliefs, Int. J. Educ. Res, ISSN: 1866-6671, 7(1), 176-197 (2015)

Merchan, N., y Hernández, N., Rol Profesoral y Estrategias Promotoras de Autorregulación del Aprendizaje en Educación Superior, Espacios, ISSN: 0798-1015, 39(52), 1-12 (2018)

Nepal, K., y Kumar, S., Teachers' Perception of the Students' Readiness for Self- Regulated Learning During the COVID-19 Pandemic, NELTA J, ISSN: 2091-0487, 25(1), 165-178 (2021)

Peel, K., Everyday Classroom Teaching Practices for Self-Regulated Learning, Issues in Educational Research, 30(1), 260-282 (2020)

Pelikan, E., Lüftenegger, M., Holzer, J., y otros tres autores, Learning During COVID-19: The Role of Self-Regulated Learning, Motivation, and Procrastination for Perceived Competence, https://doi.org/10.1007/s11618-021-01002-x, Z Erziehwiss, 24(2), 393-418 (2021)

Pintrich, P., Wolters, C., y Baxter, G., Assessing metacognition and self-regulated learning, in Issues in the Measurement of Metacognition, by G. Schraw and J. Impara, 43-97, Buros Inst of Mental, ISBN: 0-910674-45-0, Nebraska, USA, (2000)

Rivera, R., y Millán, E., First-year students' experience in Higher Education in Chile in times of COVID-19, Plann. Chang., 50(3), 209-235 (2021)

Sáez-Delgado, F., Proyecto de investigación INDIN 02/2019, Dirección de Investigación e Innovación de la UCSC, titulado "Validación de un instrumento sobre variables predictoras de los docentes universitarios en la promoción intracurricular del aprendizaje autorregulado en sus estudiantes" (2020) 
Sáez-Delgado, F., Cofré, M., y otros cinco autores, Escala de Autoeficacia Docente para la Promoción de la Autorregulación del Aprendizaje, https://doi.org/10.33210/ca.v9i3.332, CienciAmérica., 9(3), 64-68 (2020a)

Sáez-Delgado, F., Díaz-Mujica, A., Bustos, C., y Pérez-Villalobos, M., Impacto de un Programa Intracurricular Sobre la Disposición al Estudio en Universitarios, https://doi.org/10.4067/S0718-50062020000400101, Form. Univ., 13(4), 101110 (2020b)

Sáez-Delgado, F., Mella-Norambuena, J., López-Angulo, Y., y León-Ron, V., Escalas para Medir las Fases de Autorregulación del Aprendizaje en Estudiantes de Secundaria, https://doi.org/10.4067/s0718-07642021000200041, Inf. Tecnol., 32(2), 41-50 (2021)

Sáez-Delgado, F., Bustos, C., y otros cuatro autores, Disposición al Estudio, Autoeficacia y Atribuciones Causales en Estudiantes Universitarios Chilenos, https://doi.org/10.20511/pyr2018.v6n1.179, J Educ. Psychol., 6(1), 199-245 (2018)

Sava, S., Vîrgă, D., y Palos, R., The Role of Teacher Support, Students' Need Satisfaction, and Their Psychological Capital in Enhancing Students' Self-Regulated Learning, https://doi.org/10.31577/sp.2020.01.790, Stud Psychol., 62(1), 44-57 (2020)

Schober, B., Klug, J., Jöstl, G., y otros cinco autores, Gaining Substantial New Insights into University Students' SelfRegulated Learning Competencies: How Can We Succeed?, https://doi.org/10.1027/2151-2604/a00020, Z Psychol., 223(1), 64-65 (2015)

Schunk, D. H., Goal setting and self-efficacy during self-regulated learning, https://doi.org/10.1207/s15326985ep2501_6, Educ Psychol, 25(1), 71-86 (1990)

Spruce, R., y Bol, L., Teacher Beliefs, Knowledge, and Practice of Self-Regulated Learning, https://doi.org/10.1007/s11409-014-9124-0, Metacogn Learn., 10(2), 245-277 (2015)

Zimmerman, B., Attaining Self-regulation a Social Cognitive Perspective, in Handbook of Self-Regulation by M. Boekaerts, P. Pintrich, y M. Zeidner, 13-40, Elsevier Science, ISBN: 9780080575490, San Diego, USA (2000) 